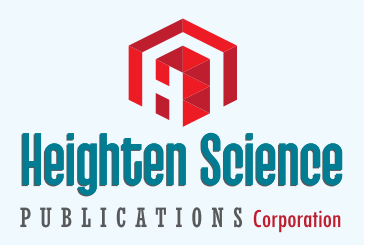

ISSN

2639-9938

\title{
A forensic treatment-monitoring study of an adult with attention- deficit disorder with hyperactivity and substance use disorder
}

\author{
Niels Peter Nielsen ${ }^{1}$ and Elisabeth H Wiig ${ }^{2 *}$ \\ 'Department of Psychiatry, Västervik Hospital, Västervik, Sweden \\ 2Department of Communication Sciences and Disorders and Knowledge Research Institute, \\ Boston University, Inc., Arlington, Texas, USA
}

\begin{abstract}
*Address for Correspondence: Elisabeth $\mathrm{H}$ Wiig Ph.D, Department of Communication Sciences and Disorders and Knowledge Research Institute, Boston University, Inc., 2131 Reflection Bay Drive, Arlington, Texas 76013, USA, Tel: +1-817-4924358; Email: ehwiig@krii.com; npn@aqt.dk

Submitted: 17 June 2019

Approved: 26 June 2019

Published: 27 June 2019

Copyright: @ 2019 Nielsen NP, et al. This is an open access article distributed under the Creative Commons Attribution License, which permits unrestricted use, distribution, and reproduction in any medium, provided the original work is properly cited
\end{abstract}

Keywords: Attention deficit disorder with hyperactivity; Substance use; Pharmacological treatment; Cognition

Check for updates

\section{Abstract}

Background: The patient in this study experienced a childhood marked by conflicts within the family, physical abuse, frequent changes of school settings, truancy, and unspecified learning disabilities. After leaving school at age 17, she was chronically depressed, had anger attacks, lacked motivation, refused psychological assistance, and had problems finding work. At age 30 , this culminated in an aggravated assault of a female in an urban public space, after which she was referred to the criminal courts. She was granted probation and hospitalized for psychiatric evaluation with follow-up, outpatient psychiatric treatment.

Aim: To present outcomes of longitudinal monitoring of methylphenidate effects on cognition and selfregulation during treatment of attention-deficit disorder with hyperactivity and substance use.

Methods: During more than a year of treatment, psychiatric interviews and norm-referenced assessments of processing-speed and cognitive overhead monitored changes in cognition and substance use.

Results: At baseline, processing-speed measures of reactive and active attention were within the averagenormal range, whereas cognitive overhead/shift cost was in the atypically high range, suggestive of ADHD symptomatology. The patient engaged in daily excessive use of cannabis, amphetamines, and other illegal substances. At the end of treatment, cognitive speed was increased and cognitive overhead normalized. With reductions in ADHD symptomatology, the patient used small amounts of cannabis during weekends and showed improved self-regulation, and legal restrictions were discontinued.

Conclusion: This forensic case provided the attending psychiatrist with clinical insights that lead to further research of methylphenidate effects on adults with ADHD and ADHD and comorbid SUD.

\section{Introduction}

This patient with early learning disabilities and early developing, undiagnosed mental health issues first came to the attention of the regional special services at age 15 , when her $8^{\text {th }}$ grade teacher referred her for psycho-educational evaluation. The referral indicated that she had been in a stable school setting during the elementary school years and had received special education services for unspecified learning disorders. During the formative years, between Grades 6 and 8, she changed schools more than five times. She was reported to be frequently truant, to experiment with cannabis, amphetamines, cocaine and other illegal substances, and to consistently defy authority. The patient provided written informed consent, according to the revised Declaration of Helsinki (World Health Organization, 2011), for us to share and publish an account of her background and treatment. The following sections will focus on the patient's family history, followed by accounts of increasing substance use and of her criminal history. 
Family History - The family history revealed that her parents divorced when she was 6 years old and that she lived with her mother and stepfather in an adversary relationship with psychological and physical abuse. The mother was a teenager at the time of her birth and her biological father and stepfather were both alcoholics. Parenting practices in the home were reported to be characterized by rigidity, physical punishment by the stepfather, reprisals in the form of house arrests and withdrawal of special privileges, and threats of being removed from the family. At age 13 , she was forcefully removed from the home and she then spent her teenage years in a variety of youth homes and institutions. Between ages 16 and 17 years, she was sent to a social institution, where she was assigned a primary teacher-counselor for the duration of her one-year residency. Her frequent violent reactions and oppositional defiance in response to parenting practices covered her lack of self-esteem, and her attempts at conflict resolution were judged to be immature. She was at that time diagnosed with a borderline personality disorder.

Substance Use - During the institutional intake interview, she reported illicit substance use that began at age 13. She admitted to using 15 Ecstasy pills, 5 grams amphetamine and cocaine, and cannabis up to seven times daily (i.e., 5-10 gr) and that she had at times been involved in drug dealing. During her stay in institutions as a teenager, her substance use escalated further, and she suffered from frequent, severe mood swings and regularly defied authority. As a result, she was evicted and social services moved her to a rental room in a single home with several male renters, some of whom were also heavy substance users.

At age 17, she acquired a steady boyfriend and they moved into a studio apartment, a relationship that lasted four years. Subsequently, she was in relationships with several men, the last of whom was reported to have raped her together with a male friend. During the various relationships, she tried to find employment and finally managed to get a substitute job as assistant care giver in a preschool setting that lasted only six months. With no job, she struggled with suicidal ideation and exhibited self-destructive behavior, such as banging her head against walls and into tables and violently scratching her face. Meanwhile, her daily substance use increased, and she admitted to stealing and drug dealing to support her habits in apparent attempts to self-medicate.

Criminal History - At age 30, the woman's mental instability and associated socialization problems culminated in aggravated assault of an elderly, when standing in line and getting impatient with that person's slowness in completing a transaction. The attack led to her arrest and to facing criminal charges in a Scandinavian regional criminal court, where she pleaded guilty and was tried. During the court procedure, it was revealed that a series of previous convictions resulted in escalating fines. As examples, at age 23 she was fined for violation of public laws concerning the use of intoxicating substances and between ages 24 and 26, she was given increasingly larger fines for violating the same public laws. Based on the documented history of socialemotional problems, past mental health issues, and psychiatric disorders, the court sentenced her to probation for up to five years and referral for psychiatric services. She was first hospitalized in a closed ward at a regional psychiatric hospital. The specified and recommended services included observation, in-depth psychiatric evaluation, and subsequent ambulatory psychiatric treatment, as deemed necessary by the regional medical authorities.

During hospitalization, interviews revealed additional information about the now 30-year-old woman. When asked if she had finished her elementary and junior high schooling, she indicated that after quitting school at age 13 , she had received youth disability benefits from age 15 and on. She had been treated for borderline personality disorder since age 17 and had received psychological counseling weekly since age 
24 years. As a result of recurring self-destructive actions, she had incurred several physical infirmities. At age 29, she was prescribed anti-psychotics (i.e., olanzapine 5 mg; chlorprothixene $150 \mathrm{mg}$ daily) and anti-depressive medication (i.e., citalopram $30 \mathrm{mg}$ daily) and a trial dosage of $90 \mathrm{mg}$ methylphenidate daily. She indicated that the medication seemed to calm her down but that she did not think the amount prescribed was large enough to meet her needs as a heavy substance user.

Observations confirmed the presence of self-destructive behaviors and psychotic episodes. Psychological and psycho-educational evaluations resulted in clinical diagnoses according to ICD-10-CM that included borderline personality disorder (F60.30), persistent depressive disorder (F34.1), substance use disorder (F19.20), unspecified attention-deficit disorder (F90.9), and specific learning disorders with impairment in reading (F81.0) and written expression (F81.81). After a threemonth hospitalization period, she was transferred to an urban psychiatric center for outpatient services with more detailed evaluation of the ADHD symptomatology and of her needs for ADHD-specific medication.

\section{Methods and Materials}

The parameters for longitudinal outpatient treatment were to further delineate the nature of a possible attention deficit/hyperactivity disorder (ADHD) and its impact on her substance use and daily life. Should further psychiatric interviews and evaluations confirm an ADHD diagnosis, the charge was to (1) provide monitoring of the effects of any prescribed medication, as indicated by quantitative measures and psychiatric interviews, (2) record any reductions in the ADHD symptomatology and substance use, and (3) achieve an appropriate level of stabilization of the ADHD symptomatology and associated behaviors. The patient was referred to a psychiatrist with extended experience in monitoring stimulant-medication dose effects in adults with ADHD and establishing individualized dose timing with a norm-referenced processing-speed test, A Quick Test of Cognitive Speed (AQT) [1,2]. The treatment was provided with informed consent according to the Declaration of Helsinki (Fortaleza, 2013) and the International Conference on Harmonization guidelines for Good Clinical Practice (UCH-GCP). The psychiatric evaluation at intake substantiated the diagnosis of ADHD and further delineated the symptomatology to reflect a combined presentation (F90.2) with comorbid general anxiety disorder (F41.1) and moderate-to-severe substance use disorder (F19.229). The patient was also evaluated by an occupational therapist, who reported evidence of impulsivity, deficits in fine motor skills, visual-spatial deficits, low stress tolerance, hyperactivity, and perseverations in actions and thoughts. During the early evaluations, the patient acted with overt aggression, constant movement, and incidences of yelling at examiners. After a period of more than one month without medication, longitudinal monitoring of methylphenidate dose effects was initiated with the AQT color, form and color-form processing-speed and cognitive overhead (shift cost) measures to obtain a baseline for continued monitoring [2]. The AQT test plate for color naming showed 40 squares in four randomized colors (black, blue, red, yellow) and for form naming 40 geometric shapes (circle, line, triangle, square) in random sequence, measuring reactive attention/perceptual speed (i.e., reaction, retrieval and response time). The third test plate featured 40 stimuli with combinations of the colors and forms, and measured active attention/cognitive speed (i.e., attention, working memory and set shifting). For the patient's age range (17-54 years), the average-normal processing and naming speed, with a cut-off point at +1 $\mathrm{SD}$ of the mean, is $25 \mathrm{~s}$ for colors, $30 \mathrm{~s}$ for forms, and $55 \mathrm{~s}$ for color-form combinations [3]. The derived cognitive overhead/shift cost measure estimates processing efficiency and is obtained by using the formula [color-form $\mathrm{s}-($ color + form $)$ ] = overhead. For the patient's age the average-normal cognitive overhead/shift cost is <+/-4 s [4]. 


\section{Results}

During the period of treatment, the patient was followed closely by the attending psychiatrist. The complete methylphenidate dose-monitoring sequence with associated behavioral observations and self-rating, covering more than a year, is documented in table 1 below. In the longitudinal record, the left-hand column specifies the approximate dates of assessment of processing-speed and overhead. This is followed by three columns labelled Color, Form, and Color-Form (C-F) in which the measured naming times in seconds (processing speed) are listed. The fifth column indicates the calculated cognitive overhead/shift value. The sixth column lists the medication status at each assessment of processing speed and cognitive overhead and the seventh provides comments regarding behavioral observations and outcomes. The last column reports the ASRS v.1.1 test scores at the time of assessment.

Table 1: Longitudinal treatment protocol for monitoring stimulant-medication dose effects with color, form, color-form processing-speed and overhead/shift cost measures in seconds.

\begin{tabular}{|c|c|c|c|c|c|c|c|}
\hline Visits & Color & Form & C-F & Over. & Medication & Patient/Psychiatrist Comments & ASRS v.1.1: \\
\hline $\begin{array}{l}\text { Baseline } \\
\text { June }\end{array}$ & 19 & 18 & 50 & +13 & None for past 36 days & $\begin{array}{c}\text { Patient uses } 30 \text { cigarettes, hash } 7-8 \mathrm{gr} \text {, and illegal } \\
\text { amphetamine daily }\end{array}$ & $\begin{array}{l}\text { Part } A=23 ; \text { Part } \\
B=48 ; \text { Total } 68\end{array}$ \\
\hline August & 17 & 16 & 32 & -1 & Concerta $72 \mathrm{mg}$ a.m. & $\begin{array}{l}\text { Hash use reduced to } 2 \mathrm{gr} / \text { week, but patient reports } \\
\text { problems with sleep }\end{array}$ & \\
\hline August & 17 & 18 & 33 & -2 & $\begin{array}{l}\text { Concerta } 54 \mathrm{mg} \text { a.m. } \\
\text { Concerta } 18 \mathrm{mg} \text { p.m. }\end{array}$ & $\begin{array}{l}\text { Patient in good mood but has short temper; sleep } \\
\text { improved slightly with evening dose }\end{array}$ & $\begin{aligned} & \text { Part } A=22 \\
& \text { Part } B=36 \\
& \text { Total }=58\end{aligned}$ \\
\hline August & 17 & 16 & 34 & +1 & $\begin{array}{l}\text { Concerta } 54 \mathrm{mg} \text { a.m. } \\
\text { Concerta } 18 \mathrm{mg} \text { p.m. }\end{array}$ & $\begin{array}{l}\text { Patient reports sleeping } 8 \mathrm{hrs} . / \mathrm{night} \text {; temper } \\
\text { improved; organization problems persist. }\end{array}$ & \\
\hline October & 14 & 15 & 34 & +5 & $\begin{array}{l}\text { Ritalin } 60 \mathrm{mg} \text { a.m., } 30 \mathrm{mg} \\
\text { noon, } 30 \mathrm{mg} \text { p.m. }\end{array}$ & $\begin{array}{l}\text { Patient functions and sleeps well; no nail biting; } \\
\text { uses } 3 \text { joints/week and no illegal amphetamine; } \\
\text { organization improved. }\end{array}$ & $\begin{aligned} \text { Part } A & =17 \\
\text { Part } B & =37 \\
\text { Total } & =54\end{aligned}$ \\
\hline November & 13 & 15 & 32 & +4 & $\begin{array}{l}\text { Ritalin } 60 \mathrm{mg} \text { a.m., } 30 \mathrm{mg} \\
\text { noon, } 30 \mathrm{mg} \text { at } 5 \text { p.m., } 30 \mathrm{mg} \\
\text { p.m. }\end{array}$ & $\begin{array}{l}\text { Patient is considered to be stabilized. The use of } \\
\text { hash has been reduced to about } 1-2 \mathrm{gr} / \text { week during } \\
\text { weekends }\end{array}$ & \\
\hline January & 15 & 16 & 32 & +1 & $\begin{array}{l}\text { Ritalin } 60 \mathrm{mg} \text { a.m., } 30 \mathrm{mg} \\
\text { noon; } 30 \mathrm{mg} \text { at } 5 \text { p.m., } 30 \mathrm{mg} \\
\text { p.m. }\end{array}$ & $\begin{array}{l}\text { Patient reports being happy, not overreacting, able to } \\
\text { watch TV several hours at a time, and sleeping well. }\end{array}$ & \\
\hline
\end{tabular}

Abbreviations: $\mathrm{C}-\mathrm{F}=$ Color-Form combinations; Over. $=$ Overhead $/$ shift cost.

Baseline -- At baseline in early June and without medication, the patient, with assistance from the attending psychiatrist for interpreting questions, obtained an ASRS v1.1 rating of 68 that was indicative of ADHD symptomatology. The AQT processing-speed measures indicate that the naming times for color, form and colorform combinations were within the average-normal range for age ages 17-54 years [3]. However, the cognitive overhead/shift cost measure was in the atypical range (i.e., $>+2 \mathrm{SD} />10 \mathrm{~s}$ ) and larger than average for healthy age peers (i.e., > +/-4s) [4]. This concurred with profiles of above average-normal cognitive speed/shift cost values that were later reported to occur in $80 \%$ or more of adults with attention-deficit disorder with hyperactivity [5-10].

Stimulant medication sequence - The record for the first assessment of processing speed in August, about four weeks after being prescribed methylphenidate (Concerta $72 \mathrm{mg}$.), indicated substantial reductions in color-form naming time from $50 \mathrm{~s}$ at baseline to 32 s (i.e., > 2 SD) and in overhead/shift cost from +13 s to -1 s (i.e., $>3$ SD). These changes indicate statistical increases in cognitive speed and processing efficiency as a result of the intake of methylphenidate $[2,3]$. The perceptual-speed measures for color and form naming, on the other hand, were only slightly reduced (i.e., less than 3 s/1 SD) with methylphenidate intake [2]. There was already evidence of a reduction in the patient's substance use, which was reported to be about four joints of hash 
(i.e., about 2 gr) per week. However, the patient reported difficulties with sleep and dose timing was therefore changed to include an early morning and an early evening dose. At the second assessment about a week later, with a $54 \mathrm{mg}$ morning dose and an additional $18 \mathrm{mg}$ dose added in the early evening, neither the processing-speed nor the overhead/shift-cost measures changed substantially. The patient reported that sleep had improved somewhat with the added dose in the early evening. The ASRS v.1.1 was re-administered and the total self-rating score was reduced by 10 points to 58 . At the third assessment, after an additional week of treatment in which the morning and evening methylphenidate doses remained the same, the patient's mood seemed improved and she appeared to have better control of her temper. She reported that she now regularly slept through the night. During the psychiatric interview, the patient also indicated that based on earlier experiences with Ritalin, this form of methylphenidate seemed to have a greater calming effect on her than what she had been experiencing with Concerta. At the patient's request, the methylphenidate prescription was changed to Ritalin and the timing of intake was distributed to occur in the morning (60 mg), at noon (30 mg), and in the early evening (30 mg).

At the fourth assessment in October, about two weeks later and after continuing with a daily intake of Ritalin (60 mg.) in the morning $30 \mathrm{mg}$ at noon, and $30 \mathrm{mg}$ in the early evening, the psychiatric interview revealed several positive behavioral changes. These included reductions in nail biting and in the use of hash to three joints weekly. She reported that the change in medication had improved her concentration and ability to organize her daily life. To the psychiatrist, she exhibited normal mood and psycho-motor tempo. The ASRS v.1.1 total point score for Part A was reduced to 17 resulting in a total score of 54 . The professional team involved in behavioral treatment recommended weekly coaching to establish greater ability to organize activities of daily living and improve her potential for financial independence.

At the fifth visit a month later in November, with an additional dose of $30 \mathrm{mg}$ Ritalin ingested in the late afternoon, as recommended by the social worker, the processing-speed and cognitive overhead/shift-cost measures were stabilized and within the average-normal range. It was recognized that the total daily dose of $150 \mathrm{mg}$ methylphenidate was at the upper limits of the recommended, but the patient reported continued well-being and felt that she was receiving an adequate total dose. The social worker for the case concurred with this statement. The patient reported that she was now smoking one or two gr hash only on weekends and that her organizational skills had improved further. The processing-speed and shift-costs measures were at consistent levels and appeared to be optimized.

At the sixth assessment, two and a half month later, the patient continued to be well functioning and in control of her medication regimen and daily life tasks. At this point, the medication consisted of a total of $150 \mathrm{mg}$ Ritalin, spaced at intervals in the morning, at noon, during the afternoon and in the early evening. The processing-speed and shift-cost measures were essentially unchanged from the previous assessment and the patient appeared in a good mood, showed good concentration, and conversed easily, and her general outlook of the future was positive. At follow-up appointments, three and nine months later, while remaining on the same medication regimen, the processing-speed and overhead/shift-cost measures were essentially unchanged. The patient reported that her attitude and well-being were at a level she had never before experienced. She was therefore judged to be stabilized and the regimen of stimulant medication was considered optimized. Accordingly, the treatment team unanimously recommended to request discontinuance of the probationary period.

Endpoint of treatment report - The final report regarding the patient was delivered to the district attorney of the criminal court at the end of two years of psychiatric and behavioral treatment. In the report to the criminal court judge, the attending 
psychiatrist, psychologist, and social worker outlined the psycho-social and cognitive improvements. They concurred in a recommendation to end the probationary period of treatment by the court-appointed psychiatrist and recommended that the patient should continue outpatient psychiatric treatment as a regular patient. A summary of the report, submitted by the attending psychiatrist, included evidence of social-behavioral and cognitive improvements, including of attainment of financial independence, is featured next: With regard to financial independence, the patient has become progressively better at handling her own finances so that she is now living and handling daily affairs independently. She is currently receiving social security and disability benefits, and these allow her to deal with the economic burdens that a chronic disability engenders. Her current ability to handle financial and other responsibilities, required for independent living, are in stark contrast with the disorganization and irresponsible behaviors and inability to handle expenses and activities of daily-living that led to insolvency before treatment. Her early reliance on illicit substances in efforts to self-medicate gradually diminished throughout the course of treatment. On intake she indicated that she used large amounts of amphetamine, cannabis, cocaine, and cigarettes on a daily basis. Her consumption of substances has now been reduced primarily to smoking one or two cigarettes daily and using a minimal amount of cannabis, less than two grams, on weekends. It is notable that the patient now shows mature insight into the implications of the ADHD condition and its effects on daily living and social behaviors, when unmedicated. She fully adheres to the medical recommendations regarding the dose and time schedule for the prescribed stimulant medication and shows complete compliance. It appears that the prescribed methylphenidate (Ritalin $150 \mathrm{mg}$ ), administered in controlled doses over the duration of the day and evening, has resulted in a complete and dramatic change in all aspects of behavior and social adaptation. It is recognized that the combined daily dosage of 150 $\mathrm{mg}$ Ritalin is at the upper limits of what is considered normal in relation to height and weight. However, the effects of the medication continue to be controlled with ongoing monitoring with psychiatric interviews and with the processing-speed assessments used during treatment.

Cognition and ADHD symptomatology - The range of ADHD symptoms expressed by the patient prior to treatment with stimulant medication indicated a severe and socially devastating condition. It was marked by extreme mood swings, hyperactivity, passive or active aggression, head banging and nail biting, open defiance of authorities, excessive substance use, and evidence of pervasive executive dysfunction. Among the symptoms of dysfunction, those associated with the central executive (attention, working memory and set shifting) were blatant and associated with difficulties in planning and organizing the environment and performing daily activities. In addition, she exhibited a high level of impulsivity and lack of inhibition with flare ups of temper and verbal outbursts and inability to sit still and stay on task for even slightly extended periods of time. At the end of treatment, all symptoms associated with moderate-tosevere forms of ADHD symptomatology were alleviated and this resulted in improved self-regulation and cognitive control. This was evidenced during the ongoing psychiatric interviews and by behavioral observations by social-service case workers. The quantitative assessments of processing speed indicate stabilization of attention, working memory and set-shifting ability (active attention), as observed in the record of significantly reduced color-form naming times (cognitive speed) and normalization of cognitive overhead (processing efficiency). With the positive changes in psychosocial behaviors, the chances of a recurrence of anti-social and aggressive behaviors are considered to be minimal.

\section{Discussion}

This was one of the first longitudinal treatment and methylphenidate-effect monitoring cases for which the attending psychiatrist was in charge and in which 
the AQT processing-speed tests were used to assess changes in reactive and active attention and processing efficiency (set-shifting). The treatment sequence with methylphenidate was associated with statistical changes in processing speed and efficiency, as measured by increased cognitive speed for color-form naming and normalization of cognitive overhead/shift cost. It was also associated with drastic, self-reported reductions in daily substance use and increased cognitive control of functions associated with daily living. This case resulted in the attending psychiatrist recognizing the possible implications of considering the size of the cognitive overhead (shift cost) as an indicator of the presence of ADHD symptomatology. At baseline, the patient's perceptual (color, form) and cognitive speed (color-form combinations) measures were well within average-normal limits based on normative data for the age range from 17 to 55 years [3]. In contrast, the cognitive overhead/shift cost measure was in the atypical/pathological range (i.e., $>+2 \mathrm{SD}$ of the mean), as compared to the norms for the age range [4]. The psychiatrist's early hypothesis led to further studies that explored whether larger than average cognitive overhead (shift cost) values might differentiate healthy adults from adults with ADHD diagnoses and psychiatric patients with and without ADHD [5-7]. The prevalence of a larger than average cognitive overhead was also compared for medication naïve and previously medicated adults with ADHD diagnosis $[5,8,9]$. Among medication naïve adults with subsequent ADHD diagnoses, the cognitive overhead/shift cost values for about $90 \%$ proved larger than expected for healthy adults (i.e., $>+/-5$ s) [4]. In later studies of previously medicated adults with ADHD, between 80 and $85 \%$ presented with larger-thanaverage overhead/shift cost values [8,9]. For this patient with ADHD and substance use disorder, longitudinal monitoring of methylphenidate effects on processing speed, ASRS v.1.1 and self-regulation and behavioral control resulted in a considerably larger than recommended daily methylphenidate dose. Later research reported that the longterm substance use by chronic drug-dependent individuals may downregulate brain dopamine systems [11]. This effect has been reported to result in a need for an up to $40 \%$ higher dose of methylphenidate for patients with ADHD-SUD than for patients with ADHD without SUD [12]. As an example, a study of adults with ADHD and amphetamine dependence, recruited from medium-security prisons in Sweden, used higher than standard maximum doses $(180 \mathrm{mg})$, and this reportedly resulted in reduced ADHD symptomatology, improved clinical conditions, and retention in treatment [12-14]. Higher than recommended methylphenidate doses were also used in a recent study of methylphenidate treatment effects among adults with ADHD-SUD, in which the AQT processing-speed tests monitored changes in reactive (color, form naming) and active (color-form naming) attention and processing efficiency (overhead/shift cost) [10]. In that study, $35 \%$ of patients experienced normalization of the active attention measure (color-form naming), whereas the average cognitive overhead/shift cost value was not significantly reduced in the group.

One of the limitations of the case study is that the psychiatric or neuropsychological test results obtained during the patient's hospitalization were not made available for inclusion in this report. A second is that at the time of the out-patient treatment, the psychiatric clinic involved did not offer assessments other than psychiatric interviews and ASRS v.1.1 ratings. In the clinic, the processing-speed measures were at that time used only by the patient's attending psychiatrist. The combined treatment protocol supports that it is important to administer all AQT tests (color, form, and color-form naming), and to evaluate cognitive overhead/shift cost, when monitoring methylphenidate or other treatment effects in adults with probable ADHD. The outcomes reported for this patient further indicated that the total daily dose of methylphenidate might need to be higher than recommended and to be distributed in controlled doses at regular intervals during the day and early evening to optimize cognitive control and level of daily functioning and promote healthy sleep. 


\section{Acknowledgement}

We express our gratitude to the patient for giving written informed consent to allow us to describe the circumstances that led to the criminal charges against her and of the psychiatric treatment sequence that followed during her probation period.

\section{References}

1. Wiig EH, Nielsen NP, Minthon L, Warkentin S. A Quick Test of Cognitive Speed (AQT): Assessment of parietal function. San Antonio. Psychological Corporation. 2002.

2. Wiig, EH, Nielsen NP, Minthon L, Warkentin S. A quick test of cognitive speed (AQT). Et kort manual Swedish. Harcourt/Psych Corp. 2005.

3. Wiig EH, Nielsen NP, Jacobson J. A quick test of cognitive speed: group patterns of aging from 15 to 85. Percept Mot Skills. 2007; 104: 1067-1075. Ref.: https://tinyurl.com/y2gxt6w4

4. Nielsen NP, Wiig EH. An additive model for relations between AQT single- and dual-dimension naming speeds. Percept Mot Skills. 2011; 11: 499-508. Ref.: https://tinyurl.com/y5fmf8yn

5. Nielsen NP, Wiig EH. Validation of the AQT color-form additive model for screening and monitoring pharmacological treatment of ADHD. J Atten Dis. 2011; 17: 187-193. Ref.: https://tinyurl.com/y38ghbs6

6. Nielsen NP, Wiig EH. AQT cognitive speed and processing efficiency differentiate adults with and without ADHD: A preliminary study. Int J Psychiatry Clin Pract. 2011; 15: 219-227. Ref.: https://tinyurl.com/y6ywodjq

7. Wiig EH, Nielsen NP. A Quick Test of Cognitive Speed for comparing processing speed and efficiency to differentiate adolescent and adult psychiatric referrals with and without attention-deficit/ hyperactivity disorders. Prim Care Companion CNS Dis. 2012; 14. Ref.: https://tinyurl.com/y3ruzhl5

8. Nielsen NP, Wiig EH, Bäck S, Gustafsson J. Processing-speed can monitor stimulant medication effects in adults with Attention Deficit Disorder with Hyperactivity. Nordic J Psychiatry. 2017; 71 296-303. Ref.: https://tinyurl.com/yy9oenjv

9. Magell G, Gustafsson J, Wiig EH, Nielsen NP. Monitoring methylphenidate dose effects in adults with attention deficit disorder with hyperactivity: A validation study. J Neuorsci Neuropsychol. 2018; 2: 104.

10. Nielsen NP, Wig EH. Review of screening and monitoring treatment of ADHD in adults with processing-speed. 2019.

11. Volkov ND, Fowler JS, Wang GJ, Swanson JM, Telang F. Dopamine in drug abuse and addiction, Arch Neurol. 2007; 64: 1575-1579. Ref.: https://tinyurl.com/yxvghfzo

12. Konstenius M, Jayaram-Lindström N, Guterstam J, Beck O, Phillips B, Franck J. Methylphenidate for ADHD and drug relapse in criminal offenders with substance dependence: A 24-week randomized placebo-controlled trial. Addiction. 2014; 109: 440-449. Ref.: https://tinyurl.com/y35nf6x5

13. Skoglund C, Brandt L, D'Onofrio B, Larsson H., Franck J. Methylphenidate doses in Attention Deficit/ Hyperactivity Disorder and comorbid substance use disorders. Eur Neuropsychopharmacol. 2017; 27: 1144-1152. Ref.: https://tinyurl.com/y63eccoz

14. Arvidsson M, Dahl ML, Franck J, Wiig EH, Nielsen NP. Methylphenidate effects on processing speed in a clinical sample of adults with ADHD and substance use disorder: a pilot study. Nordic $J$ Psychiatry. 2019; 73: 118-124. Ref.: https://tinyurl.com/y4xqw2qv 